\title{
Global attraction to solitary waves for Klein-Gordon equation with mean field interaction
}

\author{
AlexANDER KOMECH * \\ Faculty of Mathematics, University of Vienna, Wien A-1090, Austria \\ Institute for Information Transmission Problems, Moscow 101447, Russia \\ ANDREW KOMECH ${ }^{\dagger}$ \\ Mathematics Department, Texas A\&M University, College Station, TX, USA \\ Institute for Information Transmission Problems, Moscow 101447, Russia
}

March 10, 2008

\begin{abstract}
We consider a $\mathbf{U}(1)$-invariant nonlinear Klein-Gordon equation in dimension $n \geq 1$, self-interacting via the mean field mechanism. We analyze the long-time asymptotics of finite energy solutions and prove that, under certain generic assumptions, each solution converges as $t \rightarrow \pm \infty$ to the two-dimensional set of all "nonlinear eigenfunctions" of the form $\phi(x) e^{-i \omega t}$. This global attraction is caused by the nonlinear energy transfer from lower harmonics to the continuous spectrum and subsequent dispersive radiation.
\end{abstract}

\section{Introduction and main results}

In this paper, we establish the global attraction to the variety of all solitary waves for the complex Klein-Gordon field $\psi(x, t)$ with the mean field self-interaction:

$$
\left\{\begin{array}{l}
\ddot{\psi}(x, t)=\Delta \psi(x, t)-m^{2} \psi(x, t)+\rho(x) F(\langle\rho, \psi(\cdot, t)\rangle), \quad x \in \mathbb{R}^{n}, \quad n \geq 1, \quad t \in \mathbb{R}, \\
\left.\psi\right|_{t=0}=\psi_{0}(x),\left.\quad \dot{\psi}\right|_{t=0}=\pi_{0}(x),
\end{array}\right.
$$

where

$$
\langle\rho, \psi(\cdot, t)\rangle=\int_{\mathbb{R}^{n}} \bar{\rho}(x) \psi(x, t) d^{n} x .
$$

We assume that $\rho$ is a smooth real-valued function from the Schwartz class: $\rho \in \mathscr{S}\left(\mathbb{R}^{n}\right), \rho \not \equiv 0$.

The long time asymptotics for nonlinear wave equations have been the subject of intensive research, starting with the pioneering papers by Segal [Seg63a. Seg63b], Strauss [Str68], and Morawetz and Strauss [MS72], where the nonlinear scattering and the local attraction to zero solution were proved. Local attraction to solitary waves, or asymptotic stability, in $\mathbf{U}$ (1)-invariant dispersive systems was addressed in [SW90, BP93, SW92, BP95] and then developed in [PW97, SW99, Cuc01a, Cuc01b, BS03, Cuc03]. Global attraction to static, stationary solutions in dispersive systems without U(1) symmetry was first established in [Kom91, Kom95, KV96, KSK97, Kom99, KS00].

The present paper is our third result on the global attraction to solitary waves in $\mathbf{U}(1)$-invariant dispersive systems. In [KK07a], we proved such an attraction for the Klein-Gordon field coupled to one nonlinear oscillator. In [KK07b], we generalized this result for the Klein-Gordon field coupled to several oscillators. Now we are going to extend our theory to a

\footnotetext{
* Supported in part by Alexander von Humboldt Research Award (2006), by DFG grant 436 RUS 113/929/0-1, FWF grant P19138-N13, RFBR grant 07-01-00018a, and RFBR-DFG grant 08-01-91950-NNIOa.

${ }^{\dagger}$ Supported in part by Max-Planck Institute for Mathematics in the Sciences (Leipzig), Technische Universität München, and by the National Science Foundation under Grant DMS-0600863.
} 
higher dimensional setting, for the Klein-Gordon equation with the mean field interaction. This model could be viewed as a generalization of the $\delta$-function coupling [KK07a, KK07b] to higher dimensions. We follow the cairns of the approach we developed in [KK07a, KK07b]: the proof of the absolute continuity of the spectral density for large frequencies, the compactness argument to extract the omega-limit trajectories, and then the usage of the Titchmarsh Convolution Theorem to pinpoint the spectrum to just one frequency. The substantial modification is due to apparent impossibility to split off a dispersive component and to get the convergence to the attractor in the local energy norm, as in [KK07a, KK07b]; the convergence which we prove is $\varepsilon$-weaker. On the other hand, the proof of this slightly weaker convergence allows us to avoid the technique of quasimeasures, considerably shortening the argument.

We are aware of only one other recent advance [Ta007] in the field of nonzero global attractors for Hamiltonian PDEs. In that paper, the global attraction for the nonlinear Schrödinger equation in dimensions $n \geq 5$ was considered. The dispersive wave was explicitly specified using the rapid decay of local energy in higher dimensions. The global attractor was proved to be compact, but it was neither identified with the set of solitary waves nor was proved to be of finite dimension [Tao07, Remark 1.18].

Let us give the plan of the paper. In the remainder of this section, we formulate the assumptions and the results. The proof of the Main Theorem takes up Section 2 (where we analyze the absolute continuity of the spectrum for large frequencies) and Section 3 (where we select omega-limit trajectories and analyze their spectrum with the aid of the Titchmarsh Convolution Theorem). The example of a multifrequency solitary waves in the situation when $\rho$ is orthogonal to some of the solitary waves is constructed in Section 4 In Appendix $\mathrm{A}$ we give a brief sketch of the proof of the global well-posedness for equation (1.1).

\subsection{Hamiltonian structure}

We set $\Psi(t)=(\psi(x, t), \pi(x, t))$ and rewrite the Cauchy problem (1.1) in the vector form:

$$
\dot{\Psi}(t)=\left[\begin{array}{cc}
0 & 1 \\
\Delta-m^{2} & 0
\end{array}\right] \Psi(t)+\rho(x)\left[\begin{array}{c}
0 \\
F(\langle\rho, \psi(\cdot, t)\rangle)
\end{array}\right],\left.\quad \Psi\right|_{t=0}=\Psi_{0}, \quad x \in \mathbb{R}^{n}, \quad n \geq 1, \quad t \in \mathbb{R},
$$

where $\Psi_{0}=\left(\psi_{0}, \pi_{0}\right)$. We assume that the nonlinearity $F$ admits a real-valued potential:

$$
F(z)=-\nabla U(z), \quad z \in \mathbb{C}, \quad U \in C^{2}(\mathbb{C}),
$$

where the gradient is taken with respect to $\operatorname{Re} z$ and $\operatorname{Im} z$. Then equation (1.2) formally can be written as a Hamiltonian system,

$$
\dot{\Psi}(t)=J D \mathcal{H}(\Psi), \quad J=\left[\begin{array}{cc}
0 & 1 \\
-1 & 0
\end{array}\right],
$$

where $D \mathcal{H}$ is the variational derivative of the Hamilton functional

$$
\mathcal{H}(\Psi)=\frac{1}{2} \int_{\mathbb{R}^{n}}\left(|\pi|^{2}+|\nabla \psi|^{2}+m^{2}|\psi|^{2}\right) d^{n} x+U(\langle\rho, \psi\rangle), \quad \Psi=\left[\begin{array}{c}
\psi(x) \\
\pi(x)
\end{array}\right] .
$$

We assume that the potential $U(z)$ is $\mathbf{U}(1)$-invariant, where $\mathbf{U}(1)$ stands for the unitary group $e^{i \theta}, \theta \in \mathbb{R} \bmod 2 \pi$. Namely, we assume that there exists $u \in C^{2}(\mathbb{R})$ such that

$$
U(z)=u\left(|z|^{2}\right), \quad z \in \mathbb{C} .
$$

Relations (1.3) and (1.5) imply that

$$
F(z)=\alpha\left(|z|^{2}\right) z, \quad z \in \mathbb{C},
$$

where $\alpha(\cdot)=-2 u^{\prime}(\cdot) \in C^{1}(\mathbb{R})$ is real-valued. Therefore,

$$
F\left(e^{i \theta} z\right)=e^{i \theta} F(z), \quad \theta \in \mathbb{R}, \quad z \in \mathbb{C} .
$$

Due to the $\mathbf{U}(1)$-invariance, the Nöther theorem formally implies that the functional

$$
\mathcal{Q}(\Psi)=\frac{i}{2} \int_{\mathbb{R}^{n}}(\bar{\psi} \pi-\bar{\pi} \psi) d^{n} x, \quad \Psi=\left[\begin{array}{c}
\psi(x) \\
\pi(x)
\end{array}\right]
$$


is conserved for solutions $\Psi(t)$ to 1.2 .

We introduce the phase space of finite energy states for equation $(1.2)$. Denote by $\|\cdot\|_{L^{2}}$ the norm in $L^{2}\left(\mathbb{R}^{n}\right)$. Let $H^{s}\left(\mathbb{R}^{n}\right), s \in \mathbb{R}$, be the Sobolev space with the norm

$$
\|\psi\|_{H^{s}}=\left\|\left(m^{2}-\Delta\right)^{s / 2} \psi\right\|_{L^{2}} .
$$

For $s \in \mathbb{R}$ and $R>0$, denote by $H_{0}^{s}\left(\mathbb{B}_{R}^{n}\right)$ the space of distributions from $H^{s}\left(\mathbb{R}^{n}\right)$ supported in $\mathbb{B}_{R}^{n}$ (the ball of radius $R$ in $\left.\mathbb{R}^{n}\right)$. We denote by $\|\cdot\|_{H^{s}, R}$ the norm in the space $H^{s}\left(\mathbb{B}_{R}^{n}\right)$ which is defined as the dual to $H_{0}^{-s}\left(\mathbb{B}_{R}^{n}\right)$.

Definition 1.1. (i) $\mathscr{E}=H^{1}\left(\mathbb{R}^{n}\right) \oplus L^{2}\left(\mathbb{R}^{n}\right)$ is the Hilbert space of states $\Psi=\left[\begin{array}{l}\psi(x) \\ \pi(x)\end{array}\right]$, with the norm

$$
\|\Psi\|_{\mathscr{E}}^{2}=\|\pi\|_{L^{2}}^{2}+\|\nabla \psi\|_{L^{2}}^{2}+m^{2}\|\psi\|_{L^{2}}^{2}=\|\pi\|_{L^{2}}^{2}+\|\psi\|_{H^{1}}^{2} .
$$

(ii) For $\varepsilon \geq 0$, introduce the Banach spaces $\mathscr{E}^{-\varepsilon}=H^{1-\varepsilon}\left(\mathbb{R}^{n}\right) \oplus H^{-\varepsilon}\left(\mathbb{R}^{n}\right)$ with the norm defined by

$$
\|\Psi\|_{\mathscr{E}^{-\varepsilon}}^{2}=\left\|\left(m^{2}-\Delta\right)^{-\varepsilon / 2} \Psi\right\|_{\mathscr{E}}^{2}=\|\pi\|_{H^{-\varepsilon}}^{2}+\|\psi\|_{H^{1-\varepsilon}}^{2} .
$$

(iii) Define the seminorms

$$
\|\Psi\|_{\mathscr{E}^{-\varepsilon}, R}^{2}=\|\pi\|_{H^{-\varepsilon}, R}^{2}+\|\psi\|_{H^{1-\varepsilon}, R}^{2}, \quad R>0,
$$

and denote by $\mathscr{E}_{\text {loc }}^{-\varepsilon}$ the space of states $\Psi \in \mathscr{E}^{-\varepsilon}$ with finite norm

$$
\|\Psi\|_{\mathscr{E}_{\text {loc }}^{-\varepsilon}}=\sum_{R=1}^{\infty} 2^{-R}\|\Psi\|_{\mathscr{E}^{-\varepsilon}, R}<\infty
$$

We will denote $\mathscr{E}_{l o c}=\mathscr{E}_{l o c}^{0}$.

Remark 1.2. The Sobolev embedding theorem implies that the embedding $\mathscr{E} \subset \mathscr{E}_{\text {loc }}^{-\varepsilon}$ is compact for any $\varepsilon>0$.

Equation (1.2) is formally the Hamiltonian system with the phase space $\mathscr{E}$ and the Hamilton functional $\mathcal{H}$. Both $\mathcal{H}$ and $\mathcal{Q}$ are continuous functionals on $\mathscr{E}$. We introduced into 1.9 the factor $m^{2}>0$, so that $\mathcal{H}(\Psi)=\frac{1}{2}\|\Psi\|_{\mathscr{E}}^{2}+U(\langle\rho, \psi\rangle)$.

\subsection{Global well-posedness}

To have a priori estimates available for the proof of the global well-posedness, we assume that

$$
U(z) \geq A-B|z|^{2} \quad \text { for } z \in \mathbb{C}, \quad \text { where } A \in \mathbb{R} \text { and } 0 \leq B<\frac{m^{2}}{2\|\rho\|_{L^{2}}^{2}} .
$$

Theorem 1.3. Let $\rho \in \mathscr{S}\left(\mathbb{R}^{n}\right)$, and let $F(z)$ satisfy conditions (1.3), (1.5), and (1.14). Then:

(i) For every $\Psi_{0} \in \mathscr{E}$ the Cauchy problem (1.2) has a unique solution $\Psi \in C(\mathbb{R}, \mathscr{E})$.

(ii) The map $W(t): \Psi_{0} \mapsto \Psi(t)$ is continuous in $\mathscr{E}$ and $\mathscr{E}_{\text {loc }}$ for each $t \in \mathbb{R}$.

(iii) The values of the energy and charge functionals are conserved:

$$
\mathcal{H}(\Psi(t))=\mathcal{H}\left(\Psi_{0}\right), \quad \mathcal{Q}(\Psi(t))=\mathcal{Q}\left(\Psi_{0}\right), \quad t \in \mathbb{R} .
$$

(iv) The following a priori bound holds:

$$
\|\Psi(t)\|_{\mathscr{E}} \leq C\left(\Psi_{0}\right)<\infty, \quad t \in \mathbb{R}
$$

(v) For any $\varepsilon \geq 0$, the map $W(t): \Psi_{0} \mapsto \Psi(t)$ is continuous in $\mathscr{E}^{-\varepsilon}$ and $\mathscr{E}_{\text {loc }}^{-\varepsilon}$ uniformly in $t \in[-T, T]$, for any $T>0$. We sketch the proof of this theorem in Appendix $\mathrm{A}$. 


\subsection{Solitary waves}

Definition 1.4. (i) The solitary waves of equation (1.1) are solutions of the form

$$
\psi(x, t)=\phi_{\omega}(x) e^{-i \omega t}, \quad \text { where } \omega \in \mathbb{R}, \quad \phi_{\omega}(x) \in H^{1}\left(\mathbb{R}^{n}\right) .
$$

(ii) The solitary manifold is the set $\mathbf{S}=\left\{\left(\phi_{\omega},-i \omega \phi_{\omega}\right): \omega \in \mathbb{R}\right\}$, where $\phi_{\omega}$ are the amplitudes of solitary waves.

Identity 1.7 implies that the set $\mathbf{S}$ is invariant under multiplication by $e^{i \theta}, \theta \in \mathbb{R}$. Let us note that since $F(0)=0$ by 1.6), for any $\omega \in \mathbb{R}$ there is a zero solitary wave, $\phi_{\omega}(x) \equiv 0$.

Define

$$
\Sigma(x, \omega)=\mathcal{F}_{\xi \rightarrow x}\left[\frac{\hat{\rho}(\xi)}{\xi^{2}+m^{2}-\omega^{2}}\right], \quad \omega \in \mathbb{C}^{+} \cup(-m, m),
$$

where $\mathbb{C}^{+}=\{\omega \in \mathbb{C}: \operatorname{Im} \omega>0\}$. Note that $\Sigma(\cdot, \omega)$ is an analytic function of $\omega \in \mathbb{C}^{+}$with the values in $\mathscr{S}\left(\mathbb{R}^{n}\right)$. Since $|\Sigma(x, \omega)| \leq$ const $|\operatorname{Im} \omega|^{-1}$ for $\omega \in \mathbb{C}^{+}$, we can extend for any $x \in \mathbb{R}^{n}$ the function $\Sigma(x, \omega)$ to the entire real line $\omega \in \mathbb{R}$ as a boundary trace:

$$
\Sigma(x, \omega)=\lim _{\epsilon \rightarrow 0+} \Sigma(x, \omega+i \epsilon), \quad \omega \in \mathbb{R},
$$

where the limit holds in the sense of tempered distributions.

Proposition 1.5 (Existence of solitary waves). Assume that $F(z)$ satisfies 1.7 , and that $\rho \in \mathscr{S}\left(\mathbb{R}^{n}\right), \rho \not \equiv 0$. There may only be nonzero solitary wave solutions to (1.2) for $\omega \in[-m, m] \cup Z_{\rho}$, where

$$
Z_{\rho}=\left\{\omega \in \mathbb{R} \backslash[-m, m]: \hat{\rho}(\xi)=0 \text { for all } \xi \in \mathbb{R}^{n} \text { such that } m^{2}+\xi^{2}=\omega^{2}\right\} .
$$

The profiles of solitary waves are given by

$$
\hat{\phi}_{\omega}(\xi)=\frac{c \hat{\rho}(\xi)}{\xi^{2}+m^{2}-\omega^{2}}
$$

where $c \in \mathbb{C}, c \neq 0$ is a root of the equation

$$
\sigma(\omega) \alpha\left(|c|^{2}|\sigma(\omega)|^{2}\right)=1
$$

where $\alpha$ is defined in (1.6) and

$$
\sigma(\omega)=\langle\rho, \Sigma(\cdot, \omega)\rangle=\frac{1}{(2 \pi)^{n}} \int_{\mathbb{R}^{n}} \frac{|\hat{\rho}(\xi)|^{2}}{\xi^{2}+m^{2}-\omega^{2}} d^{n} \xi .
$$

The existence of such a root is a necessary condition for the existence of nonzero solitary waves (1.17).

The condition (1.21) is also sufficient for $n \geq 5$ and for $|\omega| \neq m, n \geq 1$.

For $|\omega|=m, n \leq 4$, the following additional condition is needed for sufficiency:

$$
\int_{\mathbb{R}^{n}} \frac{|\hat{\rho}(\xi)|^{2}}{\xi^{4}} d^{n} \xi<\infty
$$

Remark 1.6. As follows from 1.21 and 1.22, $\sigma(\omega)$ is strictly positive for $|\omega|<m$ (since $\rho \not \equiv 0)$ and takes finite nonzero values for all $\omega$ that correspond to solitary waves (for $n \leq 4$, the finiteness of $\sigma(\omega)$ at $\omega= \pm m$ follows if 1.23 ) is satisfied).

Remark 1.7. One can see that generically the solitary wave manifold is two-dimensional.

Proof. Substituting the ansatz $\phi_{\omega}(x) e^{-i \omega t}$ into 1.1) and using 1.6, we get the following equation on $\phi_{\omega}$ :

$$
-\omega^{2} \phi_{\omega}(x)=\Delta \phi_{\omega}(x)-m^{2} \phi_{\omega}(x)+\rho(x) F\left(\left\langle\rho, \phi_{\omega}\right\rangle\right), \quad x \in \mathbb{R}^{n} .
$$

Therefore, all solitary waves satisfy the relation

$$
\left(\xi^{2}+m^{2}-\omega^{2}\right) \hat{\phi}_{\omega}(\xi)=\hat{\rho}(\xi) F\left(\left\langle\rho, \phi_{\omega}\right\rangle\right) .
$$

For $\omega \notin[-m, m] \cup Z_{\rho}$ the relation (1.25) leads to $\phi_{\omega} \notin L^{2}\left(\mathbb{R}^{n}\right)$ (unless $\left.\phi_{\omega} \equiv 0\right)$. We conclude that there are no nonzero solitary waves for $\omega \notin[-m, m] \cup Z_{\rho}$. 
Let us consider the case $\omega \in[-m, m] \cup Z_{\rho}$. From [1.25), we see that

$$
\hat{\phi}_{\omega}(\xi)=\frac{\hat{\rho}(\xi)}{\xi^{2}+m^{2}-\omega^{2}} F\left(\left\langle\rho, \phi_{\omega}\right\rangle\right) .
$$

Using the function $\Sigma(x, \omega)$ defined in (1.18), we may express $\phi_{\omega}(x)=c \Sigma(x, \omega)$, with $c \in \mathbb{C}$. Substituting this ansatz into (1.26) and using (1.6), we can write the condition on $c$ in the form (1.21).

For $n \leq 4$, the finiteness of the energy of solitons corresponding to $\omega= \pm m$ is equivalent to the condition (1.23).

This finishes the proof of the proposition.

\subsection{The main result}

Assumption A. We assume that $\rho \in \mathscr{S}\left(\mathbb{R}^{n}\right)$, the set $Z_{\rho}$ is finite, and that

$$
\sigma(\omega) \neq 0, \quad \omega \in Z_{\rho} .
$$

Above, $Z_{\rho}$ and $\sigma(\omega)$ are defined in (1.20) and (1.22).

Remark 1.8. Note that $\sigma(\omega)$ is well-defined at the points of $Z_{\rho}$ since $\left.\hat{\rho}\right|_{|\xi|=\sqrt{\omega^{2}-m^{2}}} \equiv 0$ for $\omega \in Z_{\rho}$.

As we mentioned before, we need to assume that the nonlinearity is polynomial. This assumption is crucial in our argument: It will allow us to apply the Titchmarsh Convolution Theorem. Now all our assumptions on $F$ can be summarised as follows.

Assumption B. $F(z)$ satisfies (1.3) with the polynomial potential $U(z)$, and also satisfies (1.5) and (1.14). This can be summarised as the following assumption on $U(z)$ :

$$
U(z)=\sum_{n=1}^{p} u_{n}|z|^{2 n}, \quad u_{n} \in \mathbb{R}, \quad p \geq 2, \quad u_{p}>0 .
$$

Our main result is the following theorem.

Theorem 1.9 (Main Theorem). Assume that the coupling function $\rho(x)$ satisfies Assumption $A$ and that the nonlinearity $F(z)$ satisfies Assumption $\mathbb{B}$ Then for any $\Psi_{0} \in \mathscr{E}$ the solution $\Psi(t) \in C(\mathbb{R}, \mathscr{E})$ to the Cauchy problem (1.2) converges to $\mathbf{S}$ in the space $\mathscr{E}_{\text {loc }}^{-\varepsilon}$, for any $\varepsilon>0$ :

$$
\lim _{t \rightarrow \pm \infty} \operatorname{dist}_{\mathscr{E}_{l o c}^{-\varepsilon}}(\Psi(t), \mathbf{S})=0
$$

where $\operatorname{dist}_{\mathscr{E}_{\text {loc }}^{-\varepsilon}}(\Psi, \mathbf{S}):=\inf _{\boldsymbol{s} \in \mathbf{S}}\|\Psi-s\|_{\mathscr{E}_{\text {loc }}^{-\varepsilon}}$.

Remark 1.10. The $\mathscr{E}_{l o c}^{-\varepsilon}$-convergence to the attractor stated in this theorem is weaker than the $\mathscr{E}_{\text {loc }}$-convergence proved in [KK07a] and [KK07b], where we considered the Klein-Gordon field in dimension $n=1$, coupled to nonlinear oscillators.

Obviously, it suffices to prove Theorem 1.9 for $t \rightarrow+\infty$.

\section{Absolute continuity for large frequencies}

\subsection{Splitting of a dispersive component}

First we split the solution $\psi(x, t)$ into $\psi(x, t)=\chi(x, t)+\varphi(x, t)$, where $\chi$ and $\varphi$ are defined as solutions to the following Cauchy problems:

$$
\begin{aligned}
& \ddot{\chi}(x, t)=\Delta \chi(x, t)-m^{2} \chi(x, t),\left.\quad(\chi, \dot{\chi})\right|_{t=0}=\Psi_{0}, \\
& \ddot{\varphi}(x, t)=\Delta \varphi(x, t)-m^{2} \varphi(x, t)+\rho(x) f(t),\left.\quad(\varphi, \dot{\varphi})\right|_{t=0}=(0,0),
\end{aligned}
$$

where $\Psi_{0}$ is the initial data from $[1.2$, and

$$
f(t):=F(\langle\rho, \psi(\cdot, t)\rangle) .
$$


Note that $\langle\rho, \psi(\cdot, t)\rangle$ belongs to $C_{b}(\mathbb{R})$ since $(\psi, \dot{\psi}) \in C_{b}(\mathbb{R}, \mathscr{E})$ by Theorem $1.3(i v)$. Hence,

$$
f(\cdot) \in C_{b}(\mathbb{R}) .
$$

On the other hand, since $\chi(t)$ is a finite energy solution to the free Klein-Gordon equation, we also have

$$
(\chi, \dot{\chi}) \in C_{b}(\mathbb{R}, \mathscr{E}) .
$$

Hence, the function $\varphi(t)=\psi(t)-\chi(t)$ also satisfies

$$
(\varphi, \dot{\varphi}) \in C_{b}(\mathbb{R}, \mathscr{E}) .
$$

The following lemma reflects the well-known energy decay for the linear Klein-Gordon equation.

Lemma 2.1. There is a local decay of $\chi$ in the $\mathscr{E}_{\text {loc }}$ seminorms. That is, $\forall R>0$,

$$
\|(\chi(t), \dot{\chi}(t))\|_{\mathscr{E}, R} \rightarrow 0, \quad t \rightarrow \infty .
$$

\subsection{Complex Fourier-Laplace transform}

Let us analyze the complex Fourier-Laplace transform of $\varphi(x, t)$ :

$$
\tilde{\varphi}(x, \omega)=\mathcal{F}_{t \rightarrow \omega}[\Theta(t) \varphi(x, t)]:=\int_{0}^{\infty} e^{i \omega t} \varphi(x, t) d t, \quad \omega \in \mathbb{C}^{+}, \quad x \in \mathbb{R}^{n},
$$

where $\mathbb{C}^{+}:=\{z \in \mathbb{C}: \operatorname{Im} z>0\}$. Due to (2.6, $\tilde{\varphi}(\cdot, \omega)$ is an $H^{1}$-valued analytic function of $\omega \in \mathbb{C}^{+}$. Equation (2.2) for $\varphi$ implies that

$$
-\omega^{2} \tilde{\varphi}(x, \omega)=\Delta \tilde{\varphi}(x, \omega)-m^{2} \tilde{\varphi}(x, \omega)+\rho(x) \tilde{f}(\omega), \quad \omega \in \mathbb{C}^{+}, \quad x \in \mathbb{R}^{n},
$$

where $\tilde{f}(\omega)$ is the Fourier-Laplace transform of $f(t)$ :

$$
\tilde{f}(\omega)=\mathcal{F}_{t \rightarrow \omega}[\Theta(t) f(t)]=\int_{0}^{\infty} e^{i \omega t} f(t) d t, \quad \omega \in \mathbb{C}^{+} .
$$

The solution $\tilde{\varphi}(x, \omega)$ is analytic for $\omega \in \mathbb{C}^{+}$and can be represented by

$$
\tilde{\varphi}(x, \omega)=\Sigma(x, \omega) \tilde{f}(\omega), \quad \omega \in \mathbb{C}^{+} .
$$

\subsection{Traces of distributions for $\omega \in \mathbb{R}$}

First we remark that

$$
\Theta(t) \varphi(x, t) \in C_{b}\left(\mathbb{R}, H^{1}\left(\mathbb{R}^{n}\right)\right)
$$

by (2.6) since $\varphi(x, 0+)=0$ by initial conditions in (2.2). The Fourier-Laplace transform of $\varphi$ in time, $\mathcal{F}_{t \rightarrow \omega}[\Theta(t) \varphi(\cdot, t)]$, is a tempered $H^{1}$-valued distribution of $\omega \in \mathbb{R}$ by (2.6). We will denote this Fourier-Laplace transform by $\tilde{\varphi}(\cdot, \omega), \omega \in \mathbb{R}$, which is the boundary value of the analytic function $\tilde{\varphi}(\cdot, \omega), \omega \in \mathbb{C}^{+}$, in the following sense:

$$
\tilde{\varphi}(\cdot, \omega)=\lim _{\epsilon \rightarrow 0+} \tilde{\varphi}(\cdot, \omega+i \epsilon), \quad \omega \in \mathbb{R},
$$

where the convergence is in the space of $H^{1}$-valued tempered distributions of $\omega, \mathscr{S}^{\prime}\left(\mathbb{R}, H^{1}\left(\mathbb{R}^{n}\right)\right)$. Indeed,

$$
\tilde{\varphi}(\cdot, \omega+i \epsilon)=\mathcal{F}_{t \rightarrow \omega}\left[\Theta(t) \varphi(\cdot, t) e^{-\epsilon t}\right],
$$

while $\Theta(t) \varphi(\cdot, t) e^{-\epsilon t} \underset{\epsilon \rightarrow 0+}{\longrightarrow} \Theta(t) \varphi(\cdot, t)$, with the convergence taking place in $\mathscr{S}^{\prime}\left(\mathbb{R}, H^{1}\left(\mathbb{R}^{n}\right)\right)$ which is the space of $H^{1}$ valued tempered distributions of $t \in \mathbb{R}$. Therefore, 2.11) holds by the continuity of the Fourier transform $\mathcal{F}_{t \rightarrow \omega}$ in $\mathscr{S}^{\prime}(\mathbb{R})$. Similarly to $\left(2.11\right.$, the distribution $\tilde{f}(\omega)$ for $\omega \in \mathbb{R}$ is the boundary value of the analytic in $\mathbb{C}^{+}$function $\tilde{f}(\omega)$, $\omega \in \mathbb{C}^{+}:$

$$
\tilde{f}(\omega)=\lim _{\epsilon \rightarrow 0+} \tilde{f}(\omega+i \epsilon), \quad \omega \in \mathbb{R},
$$

since the function $\Theta(t) f(t)$ is bounded. The convergence holds in the space of tempered distributions $\mathscr{S}^{\prime}(\mathbb{R})$.

Let us justify that the representation 2.9 for $\tilde{\varphi}(x, \omega)$ is also valid when $\omega \in \mathbb{R}, \omega \neq \pm m$, if the multiplication in 2.9) is understood in the sense of distributions. 
Proposition 2.2. For any fixed $x \in \mathbb{R}^{n}, \Sigma(x, \omega), \omega \in \mathbb{R} \backslash\{ \pm m\}$, is a smooth function, and the identity

$$
\tilde{\varphi}(x, \omega)=\Sigma(x, \omega) \tilde{f}(\omega), \quad \omega \in \mathbb{R} \backslash\{ \pm m\},
$$

holds in the sense of distributions.

Proof. Consider

$$
\Sigma(x, \omega)=\frac{1}{(2 \pi)^{n}} \int_{\mathbb{R}^{n}} \frac{e^{i \xi x} \hat{\rho}(\xi) d^{n} \xi}{\xi^{2}+m^{2}-(\omega+i 0)^{2}}=\int_{0}^{\infty} \frac{R(x, \eta) d \eta}{\eta^{2}+m^{2}-(\omega+i 0)^{2}},
$$

where

$$
R(x, \eta)=\frac{1}{(2 \pi)^{n}} \int_{|\xi|=\eta} e^{i \xi x} \hat{\rho}(\xi) d^{n-1} S_{\xi} .
$$

For each $x \in \mathbb{R}^{n}, R(x, \eta)$ is smooth for $\eta>0$ and satisfies $|R(x, \eta)|=O\left(\eta^{n-1}\right)$. It follows that for each $x \in \mathbb{R}^{n}$, $\Sigma(x, \omega)$ is a smooth function of $\omega \in \mathbb{R} \backslash\{ \pm m\}$, and hence is a multiplicator in the space of distributions.

\subsection{Absolutely continuous spectrum}

Let $k(\omega)$ denote the branch of $\sqrt{\omega^{2}-m^{2}}$ such that $\operatorname{Im} \sqrt{\omega^{2}-m^{2}} \geq 0$ for $\omega \in \mathbb{C}^{+}$:

$$
k(\omega)=\sqrt{\omega^{2}-m^{2}}, \quad \operatorname{Im} k(\omega)>0, \quad \omega \in \mathbb{C}^{+} .
$$

Then $k(\omega)$ is the analytic function for $\omega \in \mathbb{C}^{+}$. We extend it to $\omega \in \overline{\mathbb{C}^{+}}$by continuity.

Proposition 2.3. The distribution $\tilde{f}(\omega+i 0), \omega \in \mathbb{R}$, is absolutely continuous for $|\omega|>m$ and satisfies

$$
\int_{|\omega|>m}|\tilde{f}(\omega)|^{2} \mathscr{M}(\omega) d \omega<\infty
$$

where $\mathscr{M}(\omega)=\frac{1}{\omega^{2}} \mathscr{R}(|k(\omega)|), \mathscr{R}(\eta)=\frac{1}{(2 \pi)^{n}} \int_{|\xi|=\eta}|\hat{\rho}(\xi)|^{2} d^{n-1} S_{\xi}, \quad \eta>0$.

Remark 2.4. The function $\mathscr{M}(\omega),|\omega|>m$, is non-negative, and its set of zeros coincides with $Z_{\rho}$ defined in (1.20).

Remark 2.5. Recall that $\tilde{f}(\omega), \omega \in \mathbb{R}$, is defined by (2.12) as the trace distribution: $\tilde{f}(\omega)=\tilde{f}(\omega+i 0)$.

Proof. We will prove that for any compact interval $I$ such that $I \cap\left([-m, m] \cup Z_{\rho}\right)=\emptyset$ the following inequality holds:

$$
\int_{I}|\tilde{f}(\omega)|^{2} \mathscr{M}(\omega) d \omega \leq C,
$$

for some constant $C>0$ which does not depend on $I$. Since there is a finite number of connected components of $\mathbb{R} \backslash\left([-m, m] \cup Z_{\rho}\right)$, this will finish the proof of the proposition. Let us prove (2.18). The Parseval identity applied to

$$
\tilde{\varphi}(x, \omega+i \epsilon)=\int_{0}^{\infty} \varphi(x, t) e^{i \omega t-\epsilon t} d t, \quad \omega \in \mathbb{R}, \quad \epsilon>0,
$$

leads to

$$
\int_{\mathbb{R}}\|\tilde{\varphi}(\cdot, \omega+i \epsilon)\|_{L^{2}}^{2} d \omega=2 \pi \int_{0}^{\infty}\|\varphi(\cdot, t)\|_{L^{2}}^{2} e^{-2 \epsilon t} d t .
$$

Since $\sup _{t>0}\|\varphi(\cdot, t)\|_{L^{2}}<\infty$ by (2.6), we may bound the right-hand side by $C_{1} / \epsilon$, with some $C_{1}>0$. Taking into account (2.9), we arrive at the key inequality

$$
\int_{\mathbb{R}}|\tilde{f}(\omega+i \epsilon)|^{2}\|\Sigma(\cdot, \omega+i \epsilon)\|_{L^{2}}^{2} d \omega \leq \frac{C_{1}}{\epsilon} .
$$

Lemma 2.6. Assume that $I$ is a compact interval such that $I \cap\left([-m, m] \cup Z_{\rho}\right)=\emptyset$. Then there exists $\epsilon_{I}>0$ such that

$$
\|\Sigma(\cdot, \omega+i \epsilon)\|_{L^{2}}^{2} \geq \frac{\mathscr{M}(\omega)}{40 \epsilon}, \quad \omega \in I, \quad 0<\epsilon \leq \epsilon_{I} .
$$


Proof. Let us compute the $L^{2}$-norm using the Fourier space representation. Since $\hat{\Sigma}(\xi, \omega+i \epsilon)=\frac{\hat{\rho}(\xi)}{\xi^{2}+m^{2}-(\omega+i \epsilon)^{2}}$, we have:

$$
\|\Sigma(\cdot, \omega+i \epsilon)\|_{L^{2}}^{2}=\frac{1}{(2 \pi)^{n}} \int_{\mathbb{R}^{n}} \frac{\left|\hat{\rho}(\xi)^{2}\right| d^{n} \xi}{\left|\xi^{2}+m^{2}-(\omega+i \epsilon)^{2}\right|^{2}}=\int_{0}^{\infty} \frac{\mathscr{R}(\eta) d \eta}{\left|\eta^{2}+m^{2}-(\omega+i \epsilon)^{2}\right|^{2}} .
$$

Let $K_{I}$ be given by

$$
K_{I}=\left\{\eta>0: \eta^{2}=\omega^{2}-m^{2}, \omega \in I\right\}
$$

We denote

$$
\eta_{\omega}=|k(\omega)| \in K_{I}
$$

Since the function $\mathscr{R}(\eta)$ is smooth and strictly positive on $K_{I}$, there exists $\epsilon_{I}>0$, satisfying

$$
\epsilon_{I}<\min \left(m, \frac{1}{2}\left|K_{I}\right|\right)
$$

so that $\mathscr{R}\left(\eta_{2}\right) \geq \frac{1}{2} \mathscr{R}\left(\eta_{1}\right)$, for all $\eta_{1}, \eta_{2} \in K_{I}$ such that $\left|\eta_{2}-\eta_{1}\right|<\epsilon_{I}$. Hence, 2.21) yields

$$
\|\Sigma(\cdot, \omega+i \epsilon)\|_{L^{2}}^{2} \geq \frac{\mathscr{R}\left(\eta_{\omega}\right)}{2} \int_{K_{I} \cap\left[\eta_{\omega}-\epsilon, \eta_{\omega}+\epsilon\right]} \frac{d \eta}{\left|\eta^{2}+m^{2}-(\omega+i \epsilon)^{2}\right|^{2}}, \quad 0<\epsilon \leq \epsilon_{I} .
$$

Estimating the integral in the right-hand side of 2.25) via the inequality

$$
\inf _{\left|\eta-\eta_{\omega}\right| \leq \epsilon} \frac{1}{\left|\eta^{2}+m^{2}-(\omega+i \epsilon)^{2}\right|^{2}}=\frac{1}{\left(2 \eta_{\omega} \epsilon+2 \epsilon^{2}\right)^{2}+4 \omega^{2} \epsilon^{2}} \geq \frac{1}{20 \epsilon^{2} \omega^{2}},
$$

where we took into account that $\eta_{\omega}^{2}=\omega^{2}-m^{2}$ and $\epsilon \leq \epsilon_{I}<m<|\omega|$, we arrive at

$$
\|\Sigma(\cdot, \omega+i \epsilon)\|_{L^{2}}^{2} \geq \frac{\mathscr{R}\left(\eta_{\omega}\right)}{40 \epsilon^{2} \omega^{2}}\left|K_{I} \cap\left[\eta_{\omega}-\epsilon, \eta_{\omega}+\epsilon\right]\right| \geq \frac{\mathscr{R}\left(\eta_{\omega}\right)}{40 \epsilon \omega^{2}}, \quad \omega \in I, \quad 0<\epsilon \leq \epsilon_{I} .
$$

The last inequality follows since by (2.23) and (2.24) either $\left[\eta_{\omega}-\epsilon, \eta_{\omega}\right] \subset K_{I}$ or $\left[\eta_{\omega}, \eta_{\omega}+\epsilon\right] \subset K_{I}$ or both.

Substituting (2.20) into 2.19), we obtain the bound

$$
\int_{I}|\tilde{f}(\omega+i \epsilon)|^{2} \mathscr{M}(\omega) d \omega \leq 40 C_{1}, \quad 0<\epsilon \leq \epsilon_{I}
$$

We conclude that the set of functions $g_{I, \epsilon}(\omega)=\tilde{f}(\omega+i \epsilon) \sqrt{\mathscr{M}(\omega)}, 0<\epsilon \leq \epsilon_{I}$, defined for $\omega \in I$, is bounded in the Hilbert space $L^{2}(I)$, and, by the Banach Theorem, is weakly compact. The convergence of the distributions (2.12) implies the weak convergence $g_{I, \epsilon} \underset{\epsilon \rightarrow 0+}{\longrightarrow} g_{I}$ in the Hilbert space $L^{2}(I)$. The limit function $g_{I}(\omega)$ coincides with the distribution $\tilde{f}(\omega) \sqrt{\mathscr{M}(\omega)}$ restricted onto $I$. This proves the bound $(2.18)$ and finishes the proof of the proposition.

\section{Nonlinear spectral analysis of omega-limit trajectories}

\subsection{Compactness argument and omega-limit trajectories}

Fix $\Psi_{0}=\left(\psi_{0}, \pi_{0}\right) \in \mathscr{E}$, and let $\psi \in C\left(\mathbb{R}, H^{1}\left(\mathbb{R}^{n}\right)\right)$ be the solution to the Cauchy problem 1.1) with the initial data $\left.(\psi, \dot{\psi})\right|_{t=0}=\Psi_{0}$. Let $t_{j}>0, j \in \mathbb{N}$ be a sequence such that $t_{j} \rightarrow \infty$.

Since $\left.(\psi, \dot{\psi})\right|_{t_{j}}$ are bounded in $\mathscr{E}$, we can pick a subsequence of $\left\{t_{j}\right\}$, also denoted $\left\{t_{j}\right\}$, such that

$$
\left.(\psi, \dot{\psi})\right|_{t_{j}} \underset{j \rightarrow \infty}{\longrightarrow} B_{0} \quad \text { in } \mathscr{E}_{l o c}^{-\varepsilon}, \text { for any } \varepsilon>0,
$$

where $B_{0}$ is some vector from $\mathscr{E}$ (see Remark 1.2). By Theorem 1.3 , there is a solution $\beta(x, t) \in C\left(\mathbb{R}, H^{1}\left(\mathbb{R}^{n}\right)\right.$ ) to 1.1 ) with the initial data $\left.(\beta, \dot{\beta})\right|_{t=0}=B_{0} \in \mathscr{E}$ :

$$
\ddot{\beta}(x, t)=\Delta \beta(x, t)-m^{2} \beta(x, t)+\rho(x) F(\langle\rho, \beta\rangle), \quad x \in \mathbb{R}^{n}, t \in \mathbb{R} ;\left.\quad(\beta, \dot{\beta})\right|_{t=0}=B_{0} \in \mathscr{E} ;
$$


this solution satisfies the bound

$$
\sup _{t \in \mathbb{R}}\|(\beta(\cdot, t), \dot{\beta}(\cdot, t))\|_{\mathscr{E}}<\infty .
$$

Let $S_{\tau}$ be the time shift operators, $S_{\tau} f(t)=f(t+\tau)$. By 3.1 and Theorem $1.3(v)$, for any $T>0$ and $\varepsilon>0$, there is the convergence

$$
S_{t_{j}}(\psi, \dot{\psi}) \underset{j \rightarrow \infty}{\longrightarrow}(\beta, \dot{\beta}) \quad \text { in } C_{b}\left([-T, T], \mathscr{E}_{\text {loc }}^{-\varepsilon}\right) .
$$

If a function $\beta(x, t)$ appears as the limit in (3.4) for some sequence $t_{j} \rightarrow \infty$, we will call it omega-limit trajectory.

To conclude the proof of Theorem 1.9, it suffices to check that every omega-limit trajectory belongs to the set of solitary waves; that is,

$$
\beta(x, t)=\phi_{\omega_{+}}(x) e^{-i \omega_{+} t}, \quad x \in \mathbb{R}^{n}, \quad t \in \mathbb{R},
$$

with some $\omega_{+} \in \mathbb{R}$.

\subsection{Compactness of the spectrum}

We denote $g(t)=F(\langle\rho, \beta(\cdot, t)\rangle)$.

Proposition 3.1. $\operatorname{supp} \tilde{g} \subset[-m, m] \cup Z_{\rho}$, where $Z_{\rho}$ is defined in 1.20 .

Proof. By Lemma2.1,

$$
\left\|\left.(\chi, \dot{\chi})\right|_{t}\right\|_{\mathscr{E}_{l o c}} \underset{t \rightarrow \infty}{\longrightarrow} 0,
$$

hence the long-time asymptotics of the solution $\psi(x, t)$ in $\mathscr{E}_{l o c}$ depends only on the singular component $\varphi(x, t)$. The convergence (3.4), together with (3.6), prove that for any $T>0$ and $\varepsilon>0$,

$$
S_{t_{j}}(\varphi, \dot{\varphi}) \underset{j \rightarrow \infty}{\longrightarrow}(\beta, \dot{\beta}), \quad \text { in } C_{b}\left([-T, T], \mathscr{E}_{l o c}^{-\varepsilon}\right) .
$$

The convergence (3.7) implies that, for any smooth compactly supported function $\alpha(x)$, there is a convergence

$$
\left\langle\alpha, \varphi\left(\cdot, t+t_{j}\right)\right\rangle \underset{j \rightarrow \infty}{\stackrel{\mathscr{S}^{\prime}}{\longrightarrow}}\langle\alpha, \beta(\cdot, t)\rangle .
$$

Due to the continuity of the Fourier transform from $\mathscr{S}^{\prime}(\mathbb{R})$ into itself, we also have

$$
\zeta(\omega)\langle\alpha, \tilde{\varphi}(\cdot, \omega)\rangle e^{-i \omega t_{j}} \underset{j \rightarrow \infty}{\stackrel{\mathscr{S}^{\prime}}{\longrightarrow}} \zeta(\omega)\langle\alpha, \tilde{\beta}(\cdot, \omega)\rangle,
$$

where $\zeta(\omega)$ is a smooth compactly supported function. Assume that $\operatorname{supp} \zeta \cap\left([-m, m] \cup Z_{\rho}\right)=\emptyset$. Then, by Proposition 2.2, we may substitute $\zeta(\omega) \tilde{\varphi}(x, \omega)$ by $\zeta(\omega) \Sigma(x, \omega) \tilde{f}(\omega)$, getting

$$
\zeta(\omega)\langle\alpha, \Sigma(\cdot, \omega)\rangle \tilde{f}(\omega) e^{-i \omega t_{j}} \underset{j \rightarrow \infty}{\stackrel{\mathscr{S}^{\prime}}{\longrightarrow}} \zeta(\omega)\langle\alpha, \tilde{\beta}(\cdot, \omega)\rangle .
$$

Since $\tilde{f}$ is locally $L^{2}$ on $\mathbb{R} \backslash\left([-m, m] \cup Z_{\rho}\right)$ by Proposition 2.3 , while $\Sigma(x, \omega)$ is smooth in $\omega \in \mathbb{R} \backslash\{ \pm m\}$ for any $x \in \mathbb{R}^{n}$, the product $\zeta(\omega)\langle\alpha, \Sigma(\cdot, \omega)\rangle \tilde{f}(\omega)$ is in $L^{1}(\mathbb{R})$. Therefore the left-hand side of (3.9) converges to zero. It follows that $\tilde{\beta}(x, \omega) \equiv 0$ for $\omega \notin[-m, m] \cup Z_{\rho}$.

\subsection{Spectral inclusion}

Proposition 3.2. $\operatorname{supp} \tilde{g} \subset \operatorname{supp}\langle\rho, \tilde{\beta}(\cdot, \omega)\rangle$.

This proposition states that the time spectrum of $g(t)=F(\langle\rho, \beta(\cdot, t)\rangle)$ is included in the time spectrum of $\langle\rho, \beta(\cdot, t)\rangle$. This spectral inclusion plays the key role in the proof of our main result (Theorem 1.9). 
Proof. By [3.7),

$$
f\left(t+t_{j}\right)=F\left(\left\langle\rho, \varphi\left(\cdot, t+t_{j}\right)\right\rangle\right) \underset{j \rightarrow \infty}{\stackrel{C_{b}([-T, T])}{\longrightarrow}} F(\langle\rho, \beta(\cdot, t)\rangle)=g(t),
$$

for any $T>0$. Using (2.13) and taking into account that $\Sigma(x, \omega)$ is smooth for $\omega \neq \pm m$, we obtain the following relation which holds in the sense of distributions:

$$
\tilde{\beta}(x, \omega)=\Sigma(x, \omega) \tilde{g}(\omega), \quad x \in \mathbb{R}^{n}, \quad \omega \in \mathbb{R} \backslash\{ \pm m\} .
$$

Taking the pairing of (3.10) with $\rho$ and using definition of $\sigma(\omega)$ (see (1.22), we get:

$$
\langle\rho, \tilde{\beta}(\cdot, \omega)\rangle=\sigma(\omega) \tilde{g}(\omega), \quad \omega \in \mathbb{R} \backslash\{ \pm m\} .
$$

First we prove Proposition 3.2 modulo the set $\omega=\{ \pm m\}$.

Lemma 3.3. $\operatorname{supp} \tilde{g} \backslash\{ \pm m\} \subset \operatorname{supp}\langle\rho, \tilde{\beta}(\cdot, \omega)\rangle$.

Proof. By Proposition 3.1 supp $\tilde{g} \subset[-m, m] \cup Z_{\rho}$. Thus, the statement of the lemma follows from (3.11) and from noticing that $\sigma(\omega)$ is smooth and positive for $\omega \in(-m, m)$ and moreover, by Assumption $\mathrm{A}$ it is nonzero on $Z_{\rho}$.

To finish the proof of Proposition 3.2 it remains to consider the contribution of $\omega= \pm m$.

Lemma 3.4. If $\omega_{0}= \pm m$ belongs to $\operatorname{supp} \tilde{g}$, then $\omega_{0} \in \operatorname{supp}\langle\rho, \tilde{\beta}\rangle$.

Proof. In the case when $\omega_{0}= \pm m$ is not an isolated point in $[-m, m] \cap \operatorname{supp} \tilde{g}$, we use (3.11) to conclude that $\omega_{0} \in$ $\operatorname{supp}\langle\rho, \tilde{\beta}\rangle$ due to positivity of $\sigma(\omega)$ for $|\omega|<m$ (which is apparent from 1.22 ).

We are left to consider the case when $\omega_{0}=m$ or $-m$ is an isolated point in $[-m, m] \cap$ supp $\tilde{g}$. We can pick an open neighbourhood $U$ of $\omega_{0}$ such that $U \cap \operatorname{supp} \tilde{g}=\left\{\omega_{0}\right\}$ since supp $\tilde{g} \in[-m, m] \cup Z_{\rho}$ and $Z_{\rho}$ is a discrete finite set. Pick $\zeta \in C_{0}^{\infty}(\mathbb{R}), \operatorname{supp} \zeta \subset U$, such that $\zeta\left(\omega_{0}\right)=1$. First we note that

$$
\zeta(\omega) \tilde{g}(\omega)=M \delta\left(\omega-\omega_{0}\right), \quad M \in \mathbb{C} \backslash\{0\},
$$

where the derivatives of the $\delta\left(\omega-\omega_{0}\right)$ are prohibited because $\check{\zeta} * g(t)$ is bounded. By $\sqrt{3.10}$, we have $U \cap \operatorname{supp}_{\omega} \tilde{\beta} \subset\left\{\omega_{0}\right\}$, hence

$$
\zeta(\omega) \tilde{\beta}(x, \omega)=\delta\left(\omega-\omega_{0}\right) b(x), \quad b \in H^{1}\left(\mathbb{R}^{n}\right) .
$$

Again, the terms with the derivatives of $\delta\left(\omega-\omega_{0}\right)$ are prohibited because $\langle\alpha, \check{\zeta} * \beta(\cdot, t)\rangle$ are bounded for any $\alpha \in C_{0}^{\infty}\left(\mathbb{R}^{n}\right)$, while the inclusion $b(x) \in H^{1}(\mathbb{R})$ is due to $\tilde{\beta} \in \mathscr{S}^{\prime}\left(\mathbb{R}, H^{1}(\mathbb{R})\right)$.

Multiplying (3.2) by $\zeta(\omega)$ and taking into account (3.12), (3.13), and the relation $\omega_{0}^{2}=m^{2}$, we see that the distribution $b(x)$ satisfies the equation

$$
0=\Delta b(x)+M \rho(x) .
$$

Therefore, $b(x) \not \equiv 0$ due to $M \neq 0$ and $\rho(x) \not \equiv 0$. Coupling (3.13) with $\rho$ and using (3.14), we get:

$$
\zeta(\omega)\langle\rho, \tilde{\beta}(\cdot, \omega)\rangle=\delta\left(\omega-\omega_{0}\right)\langle\rho, b\rangle=-\delta\left(\omega-\omega_{0}\right) \frac{\langle\Delta b, b\rangle}{M} \neq 0,
$$

since $b \in H^{1}\left(\mathbb{R}^{n}\right)$ is nonzero. This finishes the proof of Lemma 3.4

Lemmas 3.3 and 3.4 allow us to conclude that $\operatorname{supp} \tilde{g}(\omega) \subset \operatorname{supp}\langle\rho, \tilde{\beta}(\cdot, \omega)\rangle$, finishing the proof of Proposition 3.2

\subsection{The Titchmarsh argument}

Finally, we reduce the spectrum of $\gamma(t)$ to one point using the spectral inclusion from Proposition 3.2 and the Titchmarsh Convolution Theorem.

Lemma 3.5. $\langle\rho, \beta(\cdot, t)\rangle=0$ or $\operatorname{supp}\langle\rho, \tilde{\beta}(\cdot, \omega)\rangle=\left\{\omega_{+}\right\}$, for some $\omega_{+} \in[-m, m] \cup Z_{\rho}$. 
Proof. Denote

$$
\gamma(t)=\langle\rho, \beta(\cdot, t)\rangle
$$

By 1.28), $g(t):=F(\gamma(t))=-\sum_{n=1}^{p} 2 n u_{n}|\gamma(t)|^{2 n-2} \gamma(t)$. Then, by the Titchmarsh Convolution Theorem,

$$
\sup \operatorname{supp} \tilde{g}=\max _{n \in\left\{n \leq p, u_{n} \neq 0\right\}} \sup \operatorname{supp} \underbrace{(\tilde{\bar{\gamma}} * \tilde{\gamma}) * \cdots *(\tilde{\bar{\gamma}} * \tilde{\gamma})}_{n-1} * \tilde{\gamma}=p \sup \operatorname{supp} \tilde{\gamma}+(p-1) \sup \operatorname{supp} \tilde{\bar{\gamma}} \text {. }
$$

Remark 3.6. The Titchmarsh Convolution Theorem applies because supp $\tilde{\gamma} \subset[-m, m] \cup Z_{\rho}$, and hence is compact.

Noting that sup supp $\tilde{\bar{\gamma}}=-\inf \operatorname{supp} \tilde{\gamma}$, we rewrite 3.17) as

$$
\sup \operatorname{supp} \tilde{g}=\sup \tilde{\gamma}+(p-1)(\sup \operatorname{supp} \tilde{\gamma}-\inf \operatorname{supp} \tilde{\gamma})
$$

Taking into account Proposition 3.2 and 3.18, we get the following relation:

$$
\sup \operatorname{supp} \tilde{\gamma} \geq \sup \operatorname{supp} \tilde{g}=\sup \operatorname{supp} \tilde{\gamma}+(p-1)(\sup \operatorname{supp} \tilde{\gamma}-\inf \operatorname{supp} \tilde{\gamma}) \text {. }
$$

This is only possible if supp $\tilde{\gamma} \subset\left\{\omega_{+}\right\}$, for some $\omega_{+} \in[-m, m] \cup Z_{\rho}$.

\subsection{Conclusion of the proof of Theorem 1.9}

We need to prove 3.5. As follows from Lemma 3.5. $\tilde{\gamma}(\omega)$ is a finite linear combination of $\delta\left(\omega-\omega_{+}\right)$and its derivatives. As the matter of fact, the derivatives could not be present because of the boundedness of $\gamma(t):=\langle\rho, \beta(\cdot, t)\rangle$ that follows from (3.3). Therefore, $\tilde{\gamma}=2 \pi C \delta\left(\omega-\omega_{+}\right)$, with some $C \in \mathbb{C}$. This implies the following identity:

$$
\gamma(t)=C e^{-i \omega_{+} t}, \quad C \in \mathbb{C}, \quad t \in \mathbb{R} .
$$

It follows that $\tilde{g}(\omega)=2 \pi C \delta\left(\omega-\omega_{+}\right), C \in \mathbb{C}$, and the representation 3.10 implies that $\beta(x, t)=\beta(x, 0) e^{-i \omega_{+} t}$. Due to equation (3.2) and the bound $3.3, \beta(x, t)$ is a solitary wave solution. This completes the proof of Theorem 1.9

\section{Multifrequency solutions}

Now we consider the situation when Assumption $\mathrm{A}$ is violated. In this case, we show that there could exist multifrequency solutions, indicating that the set of all (one-frequency) solitary waves is only a proper subset of the global attractor.

Fix $\omega_{1} \in(m, 3 m)$. Set $\omega_{0}=\omega_{1} / 3$ and pick $\rho \in \mathscr{S}\left(\mathbb{R}^{n}\right)$ such that the following conditions are satisfied:

$$
\begin{gathered}
\left.\hat{\rho}\right|_{|\xi|=\sqrt{\omega_{1}^{2}-m^{2}}}=0, \\
\sigma\left(\omega_{1}\right):=\frac{1}{(2 \pi)^{n}} \int_{\mathbb{R}^{n}} \frac{|\hat{\rho}(\xi)|^{2} d^{n} \xi}{\xi^{2}+m^{2}-\omega_{1}^{2}}=0 .
\end{gathered}
$$

These two equalities imply that $\sigma(\omega)$ vanishes at a certain point of $Z_{\rho}$, violating Assumption $\mathrm{A}$

Lemma 4.1. There exist $a \in \mathbb{R}, b<0$ so that equation with the nonlinearity

$$
F(z)=a z+b|z|^{2} z, \quad z \in \mathbb{C},
$$

admits multifrequency solutions $\psi \in C\left(\mathbb{R}, H^{1}\right)$ of the form

$$
\psi(x, t)=\phi_{0}(x) \sin \omega_{0} t+\phi_{1}(x) \sin \omega_{1} t, \quad \omega_{0}=\frac{\omega_{1}}{3}, \quad \phi_{0}, \phi_{1} \in H^{1}\left(\mathbb{R}^{n}\right),
$$

with both $\phi_{0}$ and $\phi_{1}$ nonzero.

Proof. To make sure that the nonlinearity does not produce higher frequencies, we assume that

$$
\left\langle\rho, \phi_{1}\right\rangle=0 .
$$

Due to this assumption,

$$
F(\langle\rho, \psi\rangle)=F\left(\left\langle\rho, \phi_{0}\right\rangle \sin \omega_{0} t\right)=a\left\langle\rho, \phi_{0}\right\rangle \sin \omega_{0} t+b\left\langle\rho, \phi_{0}\right\rangle^{3} \frac{3 \sin \omega_{0} t-\sin 3 \omega_{0} t}{4} .
$$


Collecting the terms with the factors of $\sin \omega_{0} t$ and $\sin \omega_{1} t=\sin 3 \omega_{0} t$, we rewrite the equation $\ddot{\psi}=\Delta \psi-m^{2} \psi+$ $\rho F(\langle\rho, \psi\rangle)$ as two following equalities:

$$
\begin{aligned}
-\omega_{0}^{2} \phi_{0} & =\Delta \phi_{0}-m^{2} \phi_{0}+\rho(x)\left(a\left\langle\rho, \phi_{0}\right\rangle+\frac{3 b\left\langle\rho, \phi_{0}\right\rangle^{3}}{4}\right), \\
- & \omega_{1}^{2} \phi_{1}=\Delta \phi_{1}-m^{2} \phi_{1}-\rho(x) \frac{b\left\langle\rho, \phi_{0}\right\rangle^{3}}{4} .
\end{aligned}
$$

We define $\phi_{0}(x)$ by $\hat{\phi}_{0}(\xi)=\frac{\hat{\rho}(\xi)}{\xi^{2}+m^{2}-\omega_{0}^{2}}$. Since $m^{2}-\omega_{0}^{2}>0$, there is the inclusion $\phi_{1} \in H^{1}\left(\mathbb{R}^{n}\right)$. Moreover,

$$
\left\langle\rho, \phi_{0}\right\rangle=\frac{1}{(2 \pi)^{n}} \int_{\mathbb{R}^{n}} \frac{|\rho(\xi)|^{2} d^{n} \xi}{\xi^{2}+m^{2}-\omega_{0}^{2}}=\sigma\left(\omega_{0}\right)>0,
$$

due to strict positivity of $\sigma(\omega)$ for $|\omega|<m$ (see (1.22)). Hence, for any $b$ (we take $b<0$ to comply with Assumption B), we may pick $a$ such that (4.4) is satisfied. We then use (4.5) to define the function $\phi_{1}(x)$ by

$$
\hat{\phi}_{1}(\xi)=-\frac{b\left\langle\rho, \phi_{0}\right\rangle^{3}}{4} \frac{\hat{\rho}(\xi)}{\xi^{2}+m^{2}-\omega_{1}^{2}}=-\frac{b \sigma\left(\omega_{0}\right)^{3}}{4} \frac{\hat{\rho}(\xi)}{\xi^{2}+m^{2}-\omega_{1}^{2}} .
$$

Due to (4.1), $\phi_{1} \in H^{1}\left(\mathbb{R}^{n}\right)$. We are left to check that $\phi_{0}$ satisfies the assumption (4.3). Indeed, due to (4.2),

$$
\left\langle\rho, \phi_{1}\right\rangle=-\frac{b \sigma\left(\omega_{0}\right)^{3}}{4} \frac{1}{(2 \pi)^{n}} \int_{\mathbb{R}^{n}} \frac{|\hat{\rho}(\xi)|^{2} d^{n} \xi}{\xi^{2}+m^{2}-\omega_{1}^{2}}=0 .
$$

\section{A Appendix: Global well-posedness}

The global existence stated in Theorem 1.3 is obtained by standard arguments from the contraction mapping principle. To achieve this, we use the integral representation for the solutions to the Cauchy problem 1.2 :

$$
\Psi(t)=W_{0}(t) \Psi_{0}+Z[\Psi](t), \quad Z[\Psi](t):=\int_{0}^{t} W_{0}(t-s)\left[\begin{array}{c}
0 \\
\rho F(\langle\rho, \psi(\cdot, s)\rangle)
\end{array}\right] d s, \quad \Psi=\left[\begin{array}{l}
\psi \\
\pi
\end{array}\right], \quad t \geq 0 .
$$

Here $W_{0}(t)$ is the dynamical group for the linear Klein-Gordon equation which is a unitary operator in the space $\mathscr{E}^{-\varepsilon}$ for any $\varepsilon \geq 0$. The bound

$$
\left\|Z\left[\Psi_{1}\right](t)-Z\left[\Psi_{2}\right](t)\right\|_{\mathscr{E}-\varepsilon} \leq C|t| \sup _{s \in[0, t]}\left\|\Psi_{1}(s)-\Psi_{2}(s)\right\|_{\mathscr{E}-\varepsilon}, \quad C>0, \quad|t| \leq 1, \quad \varepsilon \geq 0,
$$

which holds for any two functions $\Psi_{1}, \Psi_{2} \in C(\mathbb{R}, \mathscr{E})$, shows that $Z[\psi]$ is a contraction operator in $C\left([0, t], \mathscr{E}^{-\varepsilon}\right), \varepsilon \geq 0$, if $t>0$ is sufficiently small.

The contraction mapping theorem based on the bound A.2 on the nonlinear term allows us to prove the existence and uniqueness of a local solution in $\mathscr{E}$, as well as the continuity of the map $W(t)$ (continuity with respect to the initial data). The continuity of $W(t)$ in $\mathscr{E}_{l o c}$ follows from its continuity in $\mathscr{E}$ and the finite speed of propagation.

The conservation of the values of the energy and charge functionals, $\mathcal{H}$ and $\mathcal{Q}$, is obtained by approximating the initial data in $\mathscr{E}$ with smooth initial data and using the continuity of $W(t)$ in $\mathscr{E}$. For the proof of the a priori bound (1.16), we use (1.14) to bound $\|\Psi\|_{\mathscr{E}}$ in terms of the value of the Hamiltonian:

$$
\|\Psi\|_{\mathscr{E}}^{2} \leq \frac{2 m^{2}}{m^{2}-2 B\|\rho\|_{L^{2}}^{2}}(\mathcal{H}(\Psi)-A), \quad \Psi \in \mathscr{E} .
$$

This bound allows us to extend the existence results for all times, proving the global well-posedness of (1.2) in the energy space.

Finally, the continuity of $W(t)$ in $\mathscr{E}^{-\varepsilon}$ and $\mathscr{E}_{\text {loc }}^{-\varepsilon}, \varepsilon \geq 0$, follows from the contraction mapping theorem (based on (A.2) ) and the finite speed of propagation. 


\section{References}

[BP93] V. S. Buslaev and G. S. Perel'man, Scattering for the nonlinear Schrödinger equation: states that are close to a soliton, St. Petersburg Math. J. 4 (1993), pp. 1111-1142.

[BP95] V. S. Buslaev and G. S. Perel'man, On the stability of solitary waves for nonlinear Schrödinger equations, in Nonlinear evolution equations, vol. 164 of Amer. Math. Soc. Transl. Ser. 2, pp. 75-98, Amer. Math. Soc., Providence, RI, 1995.

[BS03] V. S. Buslaev and C. Sulem, On asymptotic stability of solitary waves for nonlinear Schrödinger equations, Ann. Inst. H. Poincaré Anal. Non Linéaire 20 (2003), pp. 419-475.

[Cuc01a] S. Cuccagna, Asymptotic stability of the ground states of the nonlinear Schrödinger equation, Rend. Istit. Mat. Univ. Trieste 32 (2001), pp. 105-118 (2002), dedicated to the memory of Marco Reni.

[Cuc01b] S. Cuccagna, Stabilization of solutions to nonlinear Schrödinger equations, Comm. Pure Appl. Math. 54 (2001), pp. 1110-1145.

[Cuc03] S. Cuccagna, On asymptotic stability of ground states of NLS, Rev. Math. Phys. 15 (2003), pp. 877-903.

[KK07a] A. I. Komech and A. A. Komech, Global attractor for a nonlinear oscillator coupled to the Klein-Gordon field, Arch. Ration. Mech. Anal. 185 (2007), pp. 105-142.

[KK07b] A. I. Komech and A. A. Komech, On global attraction to quantum stationary states II. Several nonlinear oscillators coupled to massive scalar field, MPI MIS Leipzig preprint 17/2007 (2007).

[Kom91] A. I. Komech, Stabilization of the interaction of a string with a nonlinear oscillator, Mosc. Univ. Math. Bull. 46 (1991), pp. 34-39.

[Kom95] A. I. Komech, On stabilization of string-nonlinear oscillator interaction, J. Math. Anal. Appl. 196 (1995), pp. 384-409.

[Kom99] A. Komech, On transitions to stationary states in one-dimensional nonlinear wave equations, Arch. Ration. Mech. Anal. 149 (1999), pp. 213-228.

[KS00] A. Komech and H. Spohn, Long-time asymptotics for the coupled Maxwell-Lorentz equations, Comm. Partial Differential Equations 25 (2000), pp. 559-584.

[KSK97] A. Komech, H. Spohn, and M. Kunze, Long-time asymptotics for a classical particle interacting with a scalar wave field, Comm. Partial Differential Equations 22 (1997), pp. 307-335.

[KV96] A. I. Komech and B. Vainberg, On asymptotic stability of stationary solutions to nonlinear wave and KleinGordon equations, Arch. Rational Mech. Anal. 134 (1996), pp. 227-248.

[MS72] C. S. Morawetz and W. A. Strauss, Decay and scattering of solutions of a nonlinear relativistic wave equation, Comm. Pure Appl. Math. 25 (1972), pp. 1-31.

[PW97] C.-A. Pillet and C. E. Wayne, Invariant manifolds for a class of dispersive, Hamiltonian, partial differential equations, J. Differential Equations 141 (1997), pp. 310-326.

[Seg63a] I. E. Segal, The global Cauchy problem for a relativistic scalar field with power interaction, Bull. Soc. Math. France 91 (1963), pp. 129-135.

[Seg63b] I. E. Segal, Non-linear semi-groups, Ann. of Math. (2) 78 (1963), pp. 339-364.

[Str68] W. A. Strauss, Decay and asymptotics for $\square u=f(u)$, J. Functional Analysis 2 (1968), pp. 409-457.

[SW90] A. Soffer and M. I. Weinstein, Multichannel nonlinear scattering for nonintegrable equations, Comm. Math. Phys. 133 (1990), pp. 119-146.

[SW92] A. Soffer and M. I. Weinstein, Multichannel nonlinear scattering for nonintegrable equations. II. The case of anisotropic potentials and data, J. Differential Equations 98 (1992), pp. 376-390. 
[SW99] A. Soffer and M. I. Weinstein, Resonances, radiation damping and instability in Hamiltonian nonlinear wave equations, Invent. Math. 136 (1999), pp. 9-74.

[Tao07] T. Tao, A (concentration-)compact attractor for high-dimensional non-linear Schrödinger equations, Dyn. Partial Differ. Equ. 4 (2007), pp. 1-53. 